\title{
Aplicação de metodologias ativas em núcleo de educação permanente nas organizações de saúde
}

\author{
Application of active methodologies in permanent education core in health organizations \\ Aplicación las metodologías activas en el núcleo de educación permanente las \\ organizaciones de salud
}

Karla Emanuelle Silva Luz², José Batista Luz Neto², Leisson Domingues Pinheiro ${ }^{1 \star}$, Silvia Tavares de Amorim ${ }^{1,3}$.

\section{RESUMO}

Objetivo: Realizar um levantamento bibliográfico e compreender os fatores positivos que as metodologias ativas podem proporcionar para os núcleos de educação permanentes nas Organizações de Saúde. Métodos: Trata-se de uma pesquisa de natureza bibliográfica realizada por meio de uma revisão de literatura, através de buscas em bases de dados de publicações on line. Em relação ao período de busca dos documentos existentes nas bases de dados pesquisados, adotou-se como critério de inclusão as publicações existentes de janeiro de 2008 a janeiro de 2019. Resultados: As Organizações de Saúde são sistemas complexos que, para alinhamento de fluxos, protocolos, prestação de assistência qualificada, necessitam de treinamentos e capacitações. Para isto, as metodologias ativas aproximam os profissionais da saúde para sua realidade, desenvolvendo pensamentos críticos para resolução dos problemas. Considerações finais: As metodologias ativas despontam como uma ferramenta de grande importância para a educação permanente, uma vez que atua a partir de elementos do cotidiano do trabalho para formular novas estratégias e abordagens.

Palavras chaves: Educação continuada, Aprendizagem, Bases de dados.

\begin{abstract}
Objective: To carry out a bibliographic survey and understand the factors that can be used for the permanent education centers in Health Organizations. Methods: This is a bibliographic research carried out through a literature review, through searches in databases data from online publications. Regarding the period of research of existing documents in the researched databases, adopted as an inclusion criterion as existing publications from January 2008 to January 2019. Results: As Health Organizations are complex systems that, for the alignment of fees, protocols, provision of qualified assistance, use of training and capacity building. For this, as active methodologies to bring health professionals closer to their reality, developing critical thoughts for problem solving. Final considerations: As active methodologies emerge as a tool of great importance for permanent education, since it acts from elements of the daily work for new strategies and approaches.
\end{abstract}

Key words: Continuing education, Learning, Databases.

\section{RESUMEN}

Objetivo: Realizar una encuesta bibliográfica y comprender los factores que pueden utilizarse para los centros de educación permanente en organizaciones de salud Métodos: se trata de una investigación bibliográfica realizada a través de una revisión bibliográfica, mediante búsquedas en bases de datos datos de publicaciones en línea. Con respecto al período de investigación de documentos existentes en las bases de datos investigadas, adoptado como criterio de inclusión como publicaciones existentes desde enero de 2008 hasta enero de 2019. Resultados: Como las organizaciones de salud son sistemas complejos que, para la alineación de tarifas, protocolos, provisión de asistencia calificada, uso de capacitación y desarrollo de

${ }^{1}$ Centro Integrado de Inclusão e Reabilitação (CIIR/INDSH). Belém - PA. *E-mail: leisson.ona@bol.com.br

${ }^{2}$ Faculdade Inspirar - Pós-Graduação. Belém - PA.

${ }^{3}$ Universidade Federal do Pará (UFPA). Belém - PA. 
capacidades. Para ello, como metodologías activas para acercar a los profesionales de la salud a su realidad, desarrollar pensamientos críticos para la resolución de problemas. Consideraciones finales: A medida que las metodologías activas emergen como una herramienta de gran importancia para la educación permanente, ya que actúa a partir de elementos del trabajo diario para nuevas estrategias y enfoques.

Palabras clave: Educación continua, Aprendizaje, Bases de datos.

\section{INTRODUÇÃO}

As transformações ocorrem rapidamente e a educação permanente é uma estratégia de qualificação dos colaboradores. Importante tornar uma rotina e incentivar mudanças educativas nas organizações e focar na prática como fonte de conhecimento. O profissional deve atuar ativamente nesse processo e trabalhar interdisciplinar ampliando os espaços educativos (THIOLENT MJM, et al., 2011).

A saúde, como um composto amplo e complexo, ultrapassa o binômio saúde-doença por estar inserida e ser influenciada por um período, território/ambiente, sociedade, relações econômicas, culturais e interpessoais. Por tanto, dialogar sobre os sistemas de saúde partindo desta premissa, requer uma reflexão multidimensional - setorial, interprofissional e social. Não há como estruturar fluxos e funcionamento dos serviços de saúde, a partir de um modelo único, uma vez a compreensão de saúde e doença é ampla e subjetiva; se destina e é executada por indivíduos distintos; em contextos e com demandas diferentes (SALVASTANO H, 1980).

Neste sentido, os serviços de saúde enquanto um reflexo socioeconômico e cultural, tem seu funcionamento nas organizações de saúde; a serem definidas em unidades distintas a partir da complexidade dos serviços. Estas unidades, por sua vez, apresentam no seu cotidiano de funcionamento variadas demandas quanto à assistência prestada, prática profissional, fluxos e alinhamentos (ERDMANN AL, et al., 2004).

Compreendendo esta multiplicidade, novas práticas quanto ao funcionamento e capacitação profissional têm sido inseridas nas organizações de saúde, fundamentadas na Educação Permanente em Saúde, instituída no Brasil em 2003. A Secretaria da Gestão do Trabalho e da Educação na Saúde (SGTES) construiu e implementou a inédita Política Nacional de Educação Permanente em Saúde (PNEPS) firmada pela Portaria 198/2004 e Portaria 1.996/2007 (CARDOSO MLN, et al., 2017).

A PNEPS apresenta propostas de metodologias e tecnologias para o fortalecimento do Sistema Único de Saúde (SUS), por meio da valorização da pluralidade, diversidade dos saberes, produção de conhecimento inseridos nas organizações de saúde, propondo a operacionalização com os Núcleos de Educação Permanente (NEPS) (BRASIL, 2018).

Aqui citamos a Portaria no 3.194/17, que instituiu o Programa para o Fortalecimento das Práticas de Educação Permanente em Saúde no SUS, com o intuito de promover a formação e desenvolvimento dos trabalhadores, a partir dos problemas cotidianos referentes à atenção à saúde e à organização do trabalho em saúde, dentre os pontos à ressaltar: a identificação de necessidades de Educação Permanente em Saúde (EPS) dos trabalhadores e profissionais do SUS e também o fortalecimento das práticas de EPS nos estados, Distrito Federal e municípios, com o objetivo de fortalecer a qualificação dos profissionais de saúde (BRASIL, 2018).

De fato, as técnicas de ensino empregadas têm uma importância tão relevante quanto o conteúdo que transmitem ao público, as metodologias de ensino na contemporaneidade demonstram a necessidade de aprimoramento por meio de inovações. Desta forma incide sobre as metodologias ativas a busca de um embasamento incidente sobre o processo de aprendizagem que se materialize de modo dinâmico e criativo, sendo assim, tal método se distancia largamente dos limites de estratégias educacionais propostas pelo ensino considerado tradicional. (FERNANDES MA, et al.,2018).

As metodologias ativas surgem como possíveis ferramentas para construção de novos modelos de ensinoaprendizagem e se configuram como modelos educativos que estimulam o pensar crítico-reflexivo, onde os 
sujeitos participantes agem ativamente no processo de aprender-educar-refazer, além de promover uma aproximação crítica do educando com reflexões sobre os problemas e desafios da realidade, disponibilizando recursos para pesquisar problemas e aplicando soluções mais adequadas (SOBRAL FR, 2012).

Consideram-se dois atores na metodologia ativa: o professor, que deixa a função de ensinar e transforma em facilitador para o processo de aquisição do conhecimento; e o aluno que passa para o contexto dinâmico. Todo resultado disso, é um ambiente ativo, dinâmico e constitutivo para influenciar favoravelmente as percepções do educador e o educando (FARIAS PAM, et al., 2015).

A utilização destas metodologias em instituições de saúde se faz fundamental visto que, em sua maioria, as formações dos profissionais de saúde se baseiam em organizações minuciosas da assistência médica nas mais variadas especialidades, valorização do ensino centrado no ambiente hospitalar enfocando atenção curativa, individualizada e unicausal da doença - como previa o modelo biomédico de assistência à saúde. Fatores como os supracitados corroboram para a produção de um ensino dissociado do serviço e das reais necessidades do sistema de saúde (MILTRE SM, et al.,2013).

A Educação Continuada permite ao trabalhador manter, potencializar ou melhorar sua competência para que esteja compatível com o desenvolvimento de suas responsabilidades e atributos individuais. Desenvolve as potencialidades para mudanças de atitudes e comportamentos nas áreas cognitivas, afetiva e psicomotora do ser humano na perspectiva de transformação de sua prática (MÉIR MJ, et al., 2007). A Educação Permanente em Saúde corresponde à educação em serviço, quando empregado instrumentos e recursos para formação técnica submetidos a projetos de mudanças institucionais ou orientação política das ações prestadas; constrói quadros institucionais e carreiras por serviço, além de formação profissionais entre mundo do trabalho e o mundo de ensino (CECCIM RB, 2005).

Os Núcleos de Educação Permanente (NEPs) nas Organizações de Saúde tem por função o planejamento, a organização e o apoio às ações de Educação Permanente em Saúde. Desenvolvendo atividades nos eixos de: educação em serviço - com atividades de desenvolvimento dos profissionais através de cursos, palestras, seminários de capacitação interna e externa etc; educação em saúde - para usuários e acompanhantes (cita-se os processos formativos nas salas de esperas, formação de grupos de trabalho etc); integração ensino-serviço, a qual tem por objetivo integrar os profissionais de saúde (área médica, assistencial e administrativa) aos programas institucionais conforme as necessidades do serviço e do ensino; assim como, o apoio a produção científica em saúde visando a produção de conhecimento fundamentados em estudos científicos (DISTRITO FEDERAL, 2019).

Considerando isto, a contribuição deste artigo se localiza na análise acerca da articulação entre trabalho em saúde e educação no trabalho como estratégias para a transformação das práticas em saúde. Se justifica pela necessidade de entendermos o impacto do uso de metodologias ativas em Organizações de Saúde por meio dos Núcleos de Educação Permanente. O objetivo da referida pesquisa é realizar um levantamento bibliográfico de trabalhos científicos que tenham como tema a Educação Permanente nas Organizações de Saúde e analisar os fatores positivos que a metodologia ativa pode proporcionar.

\section{MÉTODOS}

A metodologia a ser utilizada neste artigo é a revisão integrativa da literatura. As revisões integrativas permitem a construção de uma análise ampliada da literatura favorecendo a discussão sobre métodos e resultados das pesquisas, assim como reflexões para futuras pesquisas (SCORSOLINI F e SANTOS MA, 2019).

Para isto, foram utilizadas algumas bases de dados online: 1) Literatura Latino-americana e do Caribe em Ciências da Saúde (LILACS); 2) Scientific Eletronic Library Online (SCIELO) e 3) Base de dados em Enfermagem (BDENF). Inicialmente foi realizada uma busca sobre a produção do conhecimento em núcleos de interesse dos artigos relacionados à aplicação de metodologia ativas em núcleos de educação permanente nas organizações de saúde. Na busca inicial foram considerados os títulos e os resumos dos artigos para seleção ampla e prováveis trabalhos de interesses, sendo destacados os resumos (artigos que não tinham 
texto acessível) e os textos completos dos artigos, utilizando como palavras chave em português "ensino", "educação permanente" e "metodologias ativas". Em relação ao período de busca dos documentos existentes nas bases de dados pesquisados, adotou-se como critério de inclusão as publicações existentes no período de janeiro de 2008 a janeiro de 2019. Todos os artigos selecionados estavam escritos em português. Como critério de exclusão foram adotadas condições de repetições dos artigos encontrados em mais de uma base de dados, que seria computado em uma única vez, visto que se trataria do mesmo artigo.

Mediante os resultados de busca, foi realizado a seleção de todos os artigos encontrados. Os dados foram analisados com auxílio de uma planilha do Microsoft Excel ${ }^{\circledR}$. E, em seguida apresentados em um quadro para melhor apresentação dos resultados da pesquisa.

\section{RESULTADOS E DISCUSSÃO}

Como resultados da pesquisa nas bases de dados online, foram encontrados 26 artigos utilizando-se os descritores citados. Destes, apenas 6 estavam relacionados à pesquisa para síntese. Os demais enfatizavam temas que não apresentavam relação com as metodologias ativas em núcleos de educação permanente nas organizações de saúde (Quadro 1).

Quadro 1 - Artigos analisados para a revisão.

\begin{tabular}{|c|c|c|}
\hline Autor/ano & Revista & Principais achados do estudo \\
\hline CONTERNO SFR, 2013. & $\begin{array}{l}\text { Revista Brasileira de } \\
\text { educação médica. }\end{array}$ & $\begin{array}{l}\text { Há uma ênfase na necessidade de mudança de ordem } \\
\text { metodológica e da incorporação de "novas } \\
\text { metodologias" como alternativas para produzir as } \\
\text { alterações desejadas e exigidas pela realidade da } \\
\text { atenção em saúde e na formação profissional. }\end{array}$ \\
\hline $\begin{array}{ll}\text { GIGANTE } & \mathrm{RL} \\
\text { CAMPOS GW, } 2016 .\end{array}$ & $\begin{array}{l}\text { Revista Brasileira de } \\
\text { educação médica. }\end{array}$ & $\begin{array}{l}\text { À pesquisa na literatura das áreas de saúde e educação } \\
\text { no período de } 1941 \text { - realização da 10 Conferência } \\
\text { Nacional de Saúde até } 2007 \text {, quando foi publicado a } \\
\text { portara } 1996 \text { GM/MS que definiu novas diretrizes e } \\
\text { estratégias para implementação da Política Nacional de } \\
\text { Educação Permanente em Saúde. }\end{array}$ \\
\hline $\begin{array}{l}\text { FERNANDES } \mathrm{M} \text {, et al., } \\
2018 .\end{array}$ & $\begin{array}{lr}\text { Revista } & \text { de } \\
\text { Enfermagem da } & \text { da } \\
\text { Universidade federal } \\
\text { de Pernambuco. }\end{array}$ & $\begin{array}{l}\text { Mostraram-se as metodologias ativas como estratégia } \\
\text { pedagógica eficaz e facilitadora para o aprimoramento } \\
\text { e a aquisição de conhecimentos para a formação } \\
\text { profissional, em específico, para o campo de atuaçáo e } \\
\text { o manejo ao usuário dependente químico. }\end{array}$ \\
\hline $\begin{array}{l}\text { CONCEICAO CV } \\
\text { MORAES MA, } 2018 .\end{array}$ & $\begin{array}{l}\text { Revista Brasileira de } \\
\text { Educação Médica. }\end{array}$ & $\begin{array}{l}\text { A aprendizagem cooperativa é eficaz em pequenos } \\
\text { grupos, traz benefícios sociais, cognitivos e } \\
\text { psicomotores, e é favorecida no âmbito de } \\
\text { metodologias ativas com educação permanente dos } \\
\text { professores. Os resultados encontrados neste estudo } \\
\text { poderiam ser úteis para outras faculdades de Medicina } \\
\text { que almejam trabalhar com métodos ativos ou mesmo } \\
\text { para as que já os utilizam com a finalidade de orientar } \\
\text { gestores e coordenadores do curso. }\end{array}$ \\
\hline $\begin{array}{l}\text { AZEVEDO CRF } \\
\text { GOMES R, } 2019 .\end{array}$ & $\begin{array}{l}\text { Interface } \\
\text { Comunicação, Saúde, } \\
\text { Educação [online]. }\end{array}$ & $\begin{array}{l}\text { As narrativas, enquanto ferramentas educacionais } \\
\text { inovadoras em educação na saúde, aprofundaram a } \\
\text { autonomia e autodeterminação, levando os sujeitos a } \\
\text { serem "autores" de sua própria vida, tão intensa e } \\
\text { transformadora que foi a "experiência do eu". }\end{array}$ \\
\hline $\begin{array}{l}\text { ROSSETTI LT, et al., } \\
2019 .\end{array}$ & $\begin{array}{l}\text { Revista Cuidado é } \\
\text { Fundamental. }\end{array}$ & $\begin{array}{l}\text { A Educação Permanente em Saúde, entendida como } \\
\text { questionamento permanente e compartilhado do } \\
\text { processo de trabalho, com vistas à transformação das } \\
\text { práticas em saúde, é uma potente ferramenta para a } \\
\text { mudança no cotidiano dos serviços, assim como na } \\
\text { gestão do serviço de saúde. }\end{array}$ \\
\hline
\end{tabular}

Fonte: Pinheiro LD, et al., 2019. 
Em decorrência das propostas de estruturas, operacionalização e metodologias inovadoras, as metodologias ativas provocam o novo. Seja pela busca para ultrapassar limites e vencer desafios nos espaços de saúde; ao mesmo tempo, que se cria vínculos ao fortalecer a importância do trabalho em equipe; sensibiliza para o que não foi expresso em palavras e desenvolve novas habilidades que permitam a todos os inseridos no contexto, perceber o funcionamento das atividades de modo sistemático e racional (AZEVEDO CRF e ROMEU G, 2019).

Um exemplo, é a metodologia do aprendizado baseado em problema (ABP). Esta metodologia propõe que as habilidades a serem apreendidas sejam organizadas em torno de um problema, havendo uma relação recíproca entre o conhecimento e o problema. O conhecimento é estimulado pelo o problema e aplicado depois da análise, teorização e estruturação - de volta ao problema. Já na aprendizagem baseada em projetos, são conduzidos um interrogatório na conclusão da experiência da aprendizagem. Aplicando no contexto profissional, esta metodologia pode ser utilizada através da discussão dos detalhes do conteúdo e estudo de casos assistenciais, tracers clínicos ou administrativos. A metodologia estimula que os profissionais reflitam e descubram resoluções, melhorias ou manutenção de forma ativa e não receptiva, apontando os temas e conclusões mais relevantes acerca das habilidades e técnicas utilizadas (ROCHA HM e LEMOS WM, 2014).

A implementação dessas metodologias nas Organizações de saúde, contribuem para otimização do processo de aprendizagem e facilita que os profissionais desenvolvam consciências críticas e sejam capazes de desenvolver relações interprofissionais para tomadas de decisões e resoluções de problemas. Esses resultados foram comprovados no estudo de revisão teórica aos acadêmicos da área de saúde que tiveram oportunidade de vivenciar essa metodologia (MELO EFF, 2019).

As metodologias ativas exprimem a necessidade de que o docente direcione o foco no protagonismo dos discentes no processo ensino-aprendizagem, com vistas a criar empatia, autonomia, encorajamento, escuta ativa e valorizando as opiniões explicitadas com o objetivo de criar um ambiente favorável ao aprendizado. Desse modo as metodologias ativas propiciam um olhar crítico que aproxima a teoria da prática e isto facilita o processo de aprendizagem, pois, quando o profissional de saúde constata, reconhece e identifica o problema, ele se interessa por ele, o examina, refletindo e relacionando com a sua história e prática profissional (FERNANDES MA, et al., 2018).

Analisando os resultados referentes a experiência de incorporação das metodologias ativas de aprendizagem e a inclusão no seu desenvolvimento profissional em cursos de pós-graduação, observado que essa metodologia contribuiu para o planejamento dos cursos e implementação de outros cursos. Importante ressaltar que alcançaram mudanças profissionais com um novo olhar do processo ensino-aprendizagem (MARIN MJS, et al., 2009).

De acordo com estudos, um curso de residência em gerência em serviços de enfermagem em Londrina, passou a utilizar como ferramenta de ensino as metodologias ativas. Foi observado que posterior a nova proposta, os estudantes passaram a ter relatos de práticas mais ativas e que os processo de mudança influenciaram não só o curso em questão, mas as demais residências e outros módulos que compõe o currículo do curso de graduação de Enfermagem (MARLI OV e JOSÉ JBC, 2007).

Outro exemplo apresentado em trabalho científico, foi o realizado na Pontifícia Universidade Católica (PUC) do Paraná, utilizando um tipo de metodologia ativa. Como resultados, foram apresentados que a metodologia acarretou benefícios imediatos de aprendizagem. Entretanto, os autores do estudo, pontuam que as metodologias não sejam apenas impostas pelos professores, mas que estes, devam e possam estar capacitados em relação a metodologia utilizada a fim de reconhecer seus benefícios e utilizá-la adequadamente (CAMPAGNOLO R, et al., 2014).

Segundo Paiva MRF (2016), as metodologias ativas colocam o aluno diante de problemas ou desafios que mobilizem o seu potencial intelectual e estudam para compreender e superar. O professor pode criar diversas estratégias para obter o máximo de benefícios com a metodologia ativa para formação do seu aluno. Isso se vale também para os profissionais de saúde. Importante alinhar o planejamento e as metodologias ativas das 
aulas à sua missão e à sua formação profissional e humana, preparando os alunos aos novos desafios da sociedade. A modificação da cultura se inicia no institucional, passa pelo docente e chega aos estudantes (ZABALZA MA, 2010).

Qualquer prática pedagógica adotada, necessita da identificação acerca das potencialidades dos alunos. Isso contribui para formação de profissionais mais qualificados e humanizados, resgaste de suas necessidades, valorização de seu contexto e individualidade, minimizando assim, as falhas e desigualdades das políticas de saúde e educação do Brasil. Tudo isso reflete na melhoria da qualidade da assistência (PRADO ML, et al., 2012).

O facilitador necessita de conhecimento quanto às metodologias adotadas com planejamento centrado no interesse do aluno e que seja de maneira estruturada para aprimorar o conhecimento. A metodologia ativa quando aplicada na sua totalidade, potencializa o contexto discutido em diversos seguimentos (MIRANDA AP, 2017). A inovação é bastante rica e abrangente do que conceito de mudanças, renovação ou a reforma. Requer uma ação persistente e melhora a prática educativa (BUSS CS e MACKEDANZ LF, 2017).

Para acompanhar as rápidas mudanças, torna-se importante a aquisição de novas capacitações e conhecimento, intensificar a capacidade de indivíduos, empresas, de aprender e transformar este aprendizado em fator de competitividade para os mesmos (LEMOS C, 2008). Ao utilizar as metodologias ativas, a educação permanente em saúde utiliza o princípio de significância, ou seja vincula a aprendizagem aos elementos que ressaltem um sentido imediato aos atores envolvidos, a partir de então, há um distanciamento do modelo de educação tradicional ao tirar do professor o título de detentor de todo o conhecimento, fazendo com que este ganhe o papel de facilitador ao trabalhar com base nas potencialidades e dificuldades ressaltadas por cada ator do processo (CONTERNO SFR, et al., 2013).

A proposta de uma aprendizagem dinâmica ao mesmo tempo em que exige uma participação ativa do educando, também exige pesquisa e comprometimento do tutor que não raramente se verá diante de imprevistos criados pela necessidade de pensamento crítico-reflexivo por parte do aluno o que gera uma quantidade variada de situações imprevistas, novas e desconhecidas, supondo que os alunos terão melhor desempenho ao se depararem diante de situações que necessitem de um raciocínio crítico do cotidiano no qual se inserem (HOFFMANN LMA e KOIFMAN L, 2013).

A citar, o município de Embu no estado de São Paulo, que relata uma experiência importante na formação dos profissionais de saúde, por meio das reciclagens de conhecimentos dos profissionais, capacitações e promoção de eventos. A Educação Permanente nos serviços de saúde vem ao encontro das necessidades de aperfeiçoar destes mecanismos, respeitando o conhecimento dos profissionais e ampliando os espaços de aprendizagem no próprio ambiente de trabalho (CAROTTA F, et al., 2009).

Estudos apontam que os trabalhadores inseridos em um processo de Educação Permanente em saúde, podem desenvolver ou potencializar habilidades quanto: a autonomia, cidadania e práticas multidimensionais. Nesse sentido, a implementação da educação permanente assim como das metodologias ativas nos serviços de saúde, se estabelecem como uma ferramenta de cuidado e investimento não somente no processo educacional, mas sobretudo, no desenvolvimento profissional. Logo, um cuidado e investimento que abrange variadas esferas no processo de trabalho (MARTINI JG, et al., 2013). Porém o processo de transformação dos profissionais e de suas práticas é complexo e profundamente social. Estes profissionais saem de sua posição de recursos humanos para sujeitos sociais em um modelo de formação, boas práticas e de gestão já implantado (TAKAO TS, et al., 2011).

Outra questão a ser pontuada é acerca do ensino superior tradicional, por reproduz conhecimentos já elaborados, ao contrário de buscar aquisições de novos conhecimentos, que podem resultar em uma inércia da crítica da teoria do conhecimento e que favorece a um comportamento que não busca a reconstrução metodológica (JUNIOR VC, 2016). Quanto aos artigos tabulados nesta revisão integrativa, ressaltamos que os autores pontuam que os resultados, em sua maioria, apresentaram que a utilização das metodologias ativas nos diversos contextos que foram inseridas apresentam: 1) a necessidade de mudanças nas metodologias de ensino em saúde, sugerindo a inserção de "novas metodologias" para as alterações 
necessárias na atenção à saúde e na formação profissional; 2) a importância da formação dos recursos humanos como uma ferramenta para consolidação dos serviços públicos de saúde; 3) que as metodologias ativas se configuram como estratégia pedagógica e facilitadora para o aprimoramento em saúde; 4) a aprendizagem cooperativa é eficaz para pequenos grupos, trazendo benefícios sociais, cognitivos e psicomotores aos envolvidos neste processo; 5) os sujeitos envolvidos no processo de aprendizagem onde foram utilizadas metodologias ativas, aprofundaram suas autonomia e autodeterminação; 6) metodologias ativas visam a transformação das práticas em saúde, por consequência, no cotidiano dos serviços prestado - à nível profissional e a quem se destina o cuidado - e na gestão.

Todas as experiências relatas em formato de artigo, apresentam sobre os benefícios estimulados pelo processo coletivo de repensar os processos de aprendizagem e, igualmente, ressaltam acerca da necessidade de estruturação e capacitação por aqueles que propõe as metodologias nos espaços acadêmicos ou nas organizações de saúde. Assim como, estimulam que outras pesquisas possam ser feitas nesta temática a fim democratizar e popularizar a educação permanente como uma prática inovadora e necessária.

\section{CONSIDERAÇÕES FINAIS}

O aprimoramento das metodologias ativas são constantes e são fundamentais os processos de estudo e capacitação permanente, com vistas a não tomar as intervenções ou propostas nos serviços obsoletos. As organizações de saúde ainda apresentam um modelo passivo biomédico no ensino e assistência, o que se configura como um fator motivador para que as práticas que estimulem o saber e fazer crítico dos profissionais passem a ser um modelo ativo de ação com vistas a um serviço mais humanizado, qualificado e condizente com as demandas dos usuários do serviço. Importante ressaltar parcerias sólidas entre as organizações de saúde e instituições de ensino para trocar mutualmente os saberes e a reconstrução metodológicas, provocando assim, inquietações e indagações no processo ensino-aprendizagem, quebrando as lógicas do ensino tradicional. A implementação destas metodologias ativas em Núcleos de Educação Permanente favorece para que os colaboradores das organizações despertem o senso crítico e interesse por determinado assunto para efetivar as melhorias dentro dos processos.

\section{REFERÊNCIAS}

1. THIOLLENT MJM, et al. Educação permanente em enfermagem em um hospital universitário. Revista da Escola de enfermagem da Universidade de São Paulo. 2011; 45(5): 1229-36.

2. SALVASTANO H. Abordagem do binômio saúde-doença e do conceito de personalidade no ecossistema. Revista de saúde pública de São Paulo. 1980; 14: 137-42.

3. AZEVEDO CRF, ROMEU G. O uso de narrativa na educação permanente em saúde: sentidos, êxitos e limites educacionais. Interface, comunicação, saúde. 2019; 23: 1-15.

4. BRASIL. Política Nacional de Educação Permanente em Saúde: o que se tem produzido para o seu fortalecimento. Ministério da Ministério da Saúde. Secretaria de Gestão do Trabalho e da Educação na Saúde. Departamento de Gestão da Educação na Saúde. Secretaria de Gestão do Trabalho e da Educação na Saúde, Departamento de Gestão da Educação na Saúde. Ministério da Saúde, 2018.

5. BUSS CS, MACKEDANZ LF. O ensino através de projetos com metodologia ativa de ensino e de aprendizagem. Revista Thema. 2017; 14(3): 122-131.

6. CARDOSO MLM, et al. A política Nacional de Educação Permanente em saúde nas escolas de saúde pública: reflexões a partir da prática. Ciência e saúde. 2017; 22(5): 1489-1500.

7. CONTERNO SFR, et al. Inovações do século passado: origens dos referenciais pedagógicos na formação profissional em saúde. Trabalho e Educação. Rio de janeiro. 2013; 11(3): 503-523.

8. ERDMANN AL, et al. As interfaces do cuidado pelo olhar da complexidade: um estudo com um grupo de pósgraduandos de enfermagem. 2004; 9(3): 411-20.

9. FERNANDES MA, et al. Metodologias ativas como ferramenta de treinamento em saúde mental. Revista de Enfermagem da UFPE. 2018; 12: 1981-8963.

10. HOFFMANN LMA, KOIFMN L.O que há de melhor em nossa perspectiva da minha vida? Physis. Rio de Janeiro. 2013; 23(2): 573-587. 
11. JUNIOR VC. Metodologia ativa na educação médica. Revista médica. 2016; 95(3): 113-121.

12. LEMOS C. Inovação na era do conhecimento. Ciência tecnologia e sociedade: $2000 ; 8,159$.

13. MELO EFF, et al. A importância da metodologia ativa de ensino e aprendizagem na educação interprofissional na área de saúde e na formação acadêmico de odontologia. Unievangélica:2019.

14. MIRANDA AP. Previsão análise crítica-reflexiva na utilização da metodologia ativa. Enfermagem Brasil. 2017; 16(3): 182-189.

15. ROCHA HM, LEMOS WM. Metodologias ativas: do que estamos falando? Base conceitual e relato de pesquisa em andamento. Simpósio pedagógico e pesquisa em educação. 2014: 2041- 201412.

16. MARIN MJS, et al. Pós-graduação multiprofissional em saúde: resultados de experiências utilizando metodologias ativas. Interface. 2010; 14(33): 331-44.

17. MARLI OV, JOSÉ JBC.A metodologia ativa na residência em gerência do curso de enfermagem da UEL. Cogitare enfermagem. 2007; 12(3): 358-64.

18. CAMPAGNOLO R, et al. Uso da abordagem Peer Instruction como metodologia ativa de aprendiz: um relato de experiência. Sigmos. 2014; 35(2): 79-87.

19. PAIVA MRF, et al. Metodologias ativas de ensino-aprendizagem: revisão integrativa. Sanare. 2016; 15(2): $145-153$.

20. PRADO ML, et al. Arco de Charles Maguerez: refletindo estratégias de metodologia ativa na formação de profissionais de saúde. Escola Ana Nery. 2012; 16(1): 172-177.

21. CAROTTA F, et al. Educação permanente em saúde: uma estratégia de gestão para pensar, refletir e construir práticas educativas e processos de trabalhos. Saúde e sociedade. 2009; 18.

22. SOBRAL FR, CAMPOS CJC. Assistência de Enfermagem na Produção. Rev Esc Enferm USP. São Paulo. 2012; 46(1): 208-218.

23. ZABALZA MA, CERDEIRINA MA. Planificación de la docência en la univerdad: elaboración de las guías docents de la materias. Narcea.2010.

24. MILTRE SM, et al. Metodologias ativas de Ensino-aprendizagem na formação professional em saúde: debates atuais. Ciência e saúde coletiva. 2013; 2133-2144.

25. FARIAS PAM, et al. Aprendizagem ativa na educação em saúde: percurso histórico e aplicações. Revista Brasileira de educação médica. 2015; 39(1): 143-158.

26. JÚNIOR VC. Metodologia ativa na educação médica. Revista médica. 2016; 95(3): 113-121.

27. MÉIR JM, et al. Percepção da educação permanente, continuada e em serviços para enfermeiros de um hospital de Ensino. 2007; 41(3): 478-494.

28. MARTINI JG, et al. Educação permanente em saúde: uma estratégia para articular Ensino e serviço. Revista da rede de enfermagem do nordeste. 2013; 14(5): 1050-1060.

29. TAKAO TS, et al. Percepção dos médicos sobre o curso de facilitadores de educação permanente em saúde. Revista Brasileira de Educação Médica. 2011; 35(1): 132-141.

30. CECCIN RB. Educação Permanente em saúde: desafios ambiciosos e necessário. Comunicação, saúde, educação. 2005; 9(16): 161-177.

31. DISTRITO FEDERAL. Plano de educação permanente em saúde. Gerência de educação em saúde. Escola de aperfeiçoamento do SUS. 2019.

32. SCORSOLINI-COMIN F, et al. Satisfação conjugal: revisão integrativa da literatura científica nacional. Psicologia: terapia e pesquisa. 2010; 26(3): 525-531. 